\title{
Mental health and psychosocial support response in Sri Lanka during pandemic COVID-19
}

\section{Enoka Priyangani Wickramasinghe", Rohan Ratnayake, Yamuna Ellawala}

Directorate of Mental Health, Ministry of Health, Sri Lanka

"Correspondence: enoka7@gmail.com

iDhttps://orcid.org/0000-0003-2129-5496

DOI: https://doi.org/10.4038/jccpsl.v26i5.8320

Received on 8 May 2020

Accepted on 5 June 2020

\section{Summary}

With rapid spread of the infection across geographical boundaries, the high risk of mortality and the nature of control measures, the pandemic COVID-19 brought fear, anxiety and uncertainty in every global citizen including Sri Lankans irrespective of the socio-economic status, race or religion. Following the epidemic alerts from Wuhan, China (1) and World Health Organization (WHO) (2), Sri Lanka followed stringent procedures to contain the pandemic in the country. Due to factors such as fear of infection and losing loved ones, staying quarantined, prolonged curfew with social isolation, losing livelihood, closure of schools and social stigma, a wide range of psychosocial issues at all levels were anticipated (3). Also, relapses and worsening of the existing psychological conditions were foreseen (4). This background warranted Mental Health and Psycho-Social Support (MHPSS) to be prioritized (3-6,) and integrated within the 'Whole-ofGovernment and Whole-of-Society' approach in Sri Lankan COVID-19 response. Directorate of Mental Health (DMH) in Ministry of Health of Sri Lanka took the lead in planning and providing necessary MHPSS in the country. Many government and non-government key stakeholders collaborated with DMH in MHPSS during COVID-19. In addition, psychological and counselling experts embarked on providing psychological support for defined target audiences. This narrative report describes these initiatives in detail.

\section{Public health response and its impact}

\section{Implementation of MHPSS strategies}

Planning and implementation of the COVID-19 MHPSS response of Sri Lanka were done by the De Soysa Maternity Hospital (DMH) according to the guiding principles of the Mental Health Policy of Sri Lanka: treatment, rehabilitation and prevention of mental health conditions and promotion of mental wellbeing of all citizens in the country (7). Best practices from elsewhere were also referred for focused COVID-19 response (1, 3, 5-6). Major barriers for rapid action were lack of staff including consultant community physicians (CCPs) at DMH.

The MHPSS in COVID-19 by the DMH in Sri Lanka took the following path. 
1. Continuation of the management of persons with existing mental health condition/ psychiatry illness

- Continuous supply of medicines for persons followed-up at psychiatry/mental health clinics

People who are already having a mental illness are at higher risk of relapse and suffer from symptoms of acute distress during any catastrophe (3-4). All the psychiatry and mental health units in the country were advised on continuation of the management by two circulars (DMH/D/08/2020 and DDG/NCD/ 70/2020). Oral medications for these persons were arranged to be posted for two consecutive months, and injectable medicines were administered either at the community or clinics (8). Psychiatry and medical officer mental health teams with community psychiatry nursing officers did home visits for most non-compliant and vulnerable clients to administer injectable medicines. Support of the existing wellestablished public health care system was also obtained in this endeavour where necessary.

\section{- Distant psychological support}

Psychological support provided via an already established helpline of the National Institute of Mental Health (NIMH) (1926) continued. In addition, the Sri Lanka College of Psychiatrists initiated another helpline (0712578578) to support persons diagnosed with a psychiatry illness. Prolong curfew constrained hospital visits and private consultations. Hence, these distant support mechanisms helped continued psychological support mainly for persons who already diagnosed as having a mental health condition.

\section{Management of addiction to alcohol and other addictive substances}

With the disruption of supply chains of alcohol and other addictive substances, an increased number of persons seeking medical services for withdrawal symptoms were seen (4). Two sub-committees were appointed to oversee this emerged issue, support these users for recovery and to establish regular systems of registration/prescription/dispensing with adequate monitoring. Unseen underlying forces impose threats and barriers for sustained control measures and breaking supply chains which is the ultimate solution for this issue in Sri Lanka.

\section{Promotion of mental wellbeing and prevention of future mental health conditions}

- Promoting mental wellbeing of frontline healthcare staff

Healthcare staff looking after COVID-19 patients and the public health staff involved in contact tracing and quarantine processes are at the highest risk of stress and burnout (4). In response, guidance to promote mental wellbeing of frontline healthcare staff was developed by the National Technical Committee on Mental Health and DMH. This provided guidance for health administrators to implement activities to promote mental wellbeing of the frontline health staff. All hospitals have taken initiatives to promote wellbeing of the staff, in line with this guideline. Furthermore, messages and video clips were produced and disseminated by the DMH in collaboration with the Health Promotion Bureau (HPB), to facilitate mental wellbeing of health and other frontline staff.

- Promoting mental wellbeing of general public

Guidance for media - The DMH prepared a guideline for media and circulated it under the guidance of Deputy Director General of Public Health Services (DDG/PHS)-II emphasising the importance of using correct terminology and communication methods in reporting statistics pertaining to the COVID-19 situation, limitation of upsetting news, broadcasting of mental wellbeing programmes in media and responsibility of the media on preventing social stigma. Following these initiatives, many changes in media reporting resulted, warranting a well-formulated media guidance in disasters in the country. 
Using mass media to promote mental wellbeing The CCPs of DMH took the initiative to address the general-public via mass media on strategies and importance of initiating practices which promote psychological well-being and emotional balance and on available mental health services in the country (Figure 1).

Messages and video clips - The DMH and HPB developed and shared mental health promoting messages via print and electronic media with the support of experts in psychology. Video clips (Figure 2) and social media posts were used to promote mental well-being of the public. The constructive comments reflected the positive impact and value of well-planned communication campaigns for promoting mental wellbeing of people

Prevention of stigma - Due to the nature of control measures used to contain COVID-19 and the communication modalities used during this period, it created undue stigmatized behaviour among the general public. The DMH launched a rapid online survey (Figure 3) to explore the magnitude and nature of stigma and discrimination related to COVID-19, to develop an evidence-based communication campaign to combat this detrimental behaviour of people.

The interim analysis reflected high proportions of stigma towards health staff and other frontline workers especially by the society and at places where they buy essential goods.

Helplines - In addition to 1926 of NIMH, the Courage Compassion Commitment Foundation continued their helpline, 1333 to provide emotional support for the public. Moreover, 1999 helpline of the HPB also started receiving a massive number of calls complaining of distress and anxiety. In response, the DMH is currently working with Sri Lanka Telecommunications on extending the Hotline 1926 with automated diversion to the provinces where the client resides to be answered by the medical officers of mental health (MOMHs) in provinces.

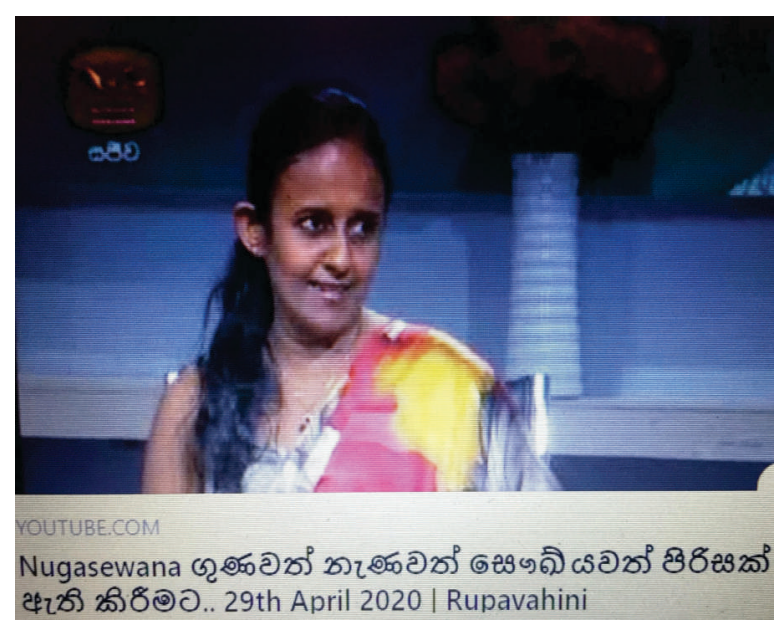

Figure 1:

Promoting emotional intelligence for psychological wellbeing
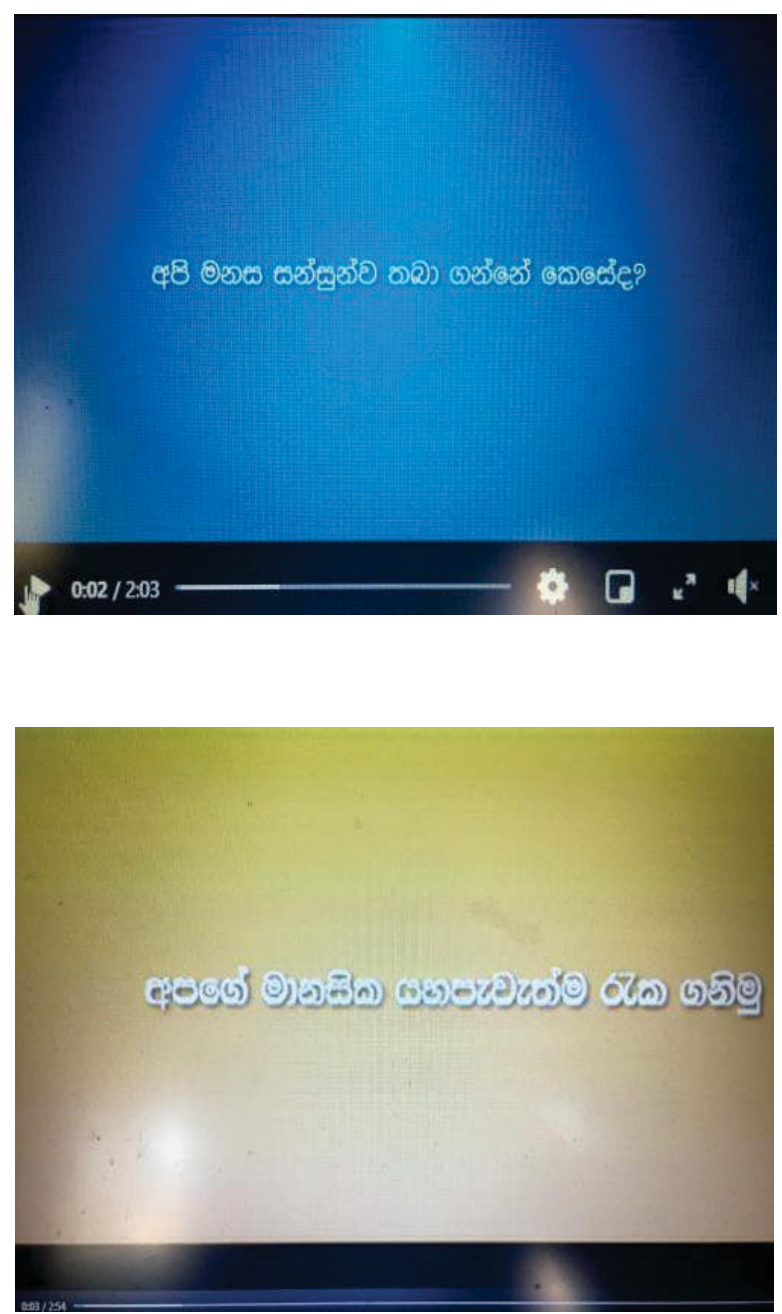

Figure 2:

Video clips to promote mental health during COVID-19 


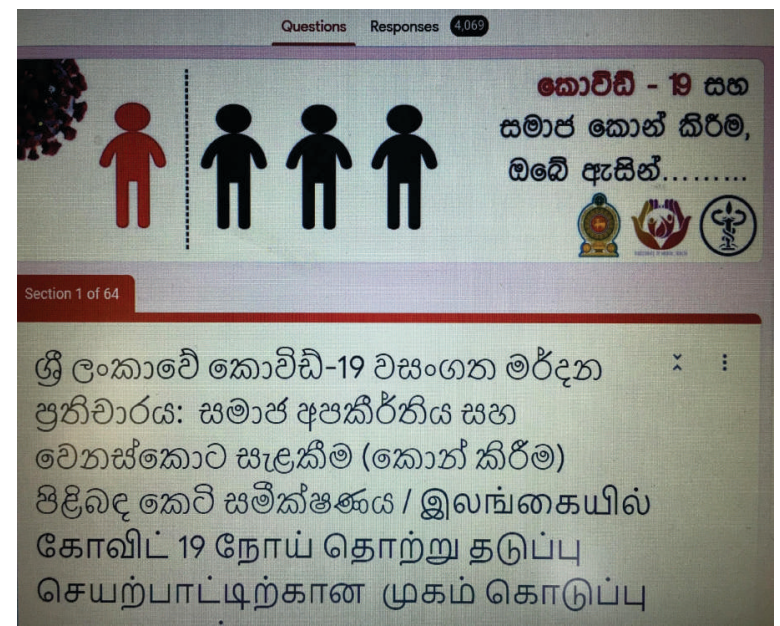

Figure.3: Online survey to assess stigma during COVID-19

4. Capacity building and strengthening the network of MOMHs for quality mental health services across country

The DMH has a network of approximately 180 MOMHs across all the districts in Sri Lanka and another $19 \mathrm{MOMH}$ (Focal Point) attached to the offices of regional directors of health services (RDHS). While providing care for persons with mental illnesses under the technical guidance of local psychiatrists, MOMHs are also engaged in promoting mental health and preventative activities in the districts. Foreseeing the constraints on inhouse training due to requirement of physical distancing to be continued for at least another 2-3 years due to COVID-19, DMH with the support of WHO is planning to develop an online distant training platform to train this vital district level mental health staff to continue strengthening mental health services in Sri Lanka.

5. Addressing social determinants of mental health of people

The Sri Lankan Government along with the Ministries of Health and Defence and all other essential service suppliers were constantly focusing on providing the basic needs of people in the country which has laid the foundation for mental health and wellbeing of people.
6. Planning for post COVID-19 resumption and normalizing of mental health services in Sri Lanka

Unlike any other disaster or catastrophe, all nations will have to live with COVID-19 preventive measures for some time, necessitating proactive planning for normalization and resumption of services at a better resilient state. The DMH has taken proactive steps for such plans, with the hope of reaching a better level in providing mental health services for citizens of Sri Lanka, with the support of other key stakeholders.

\section{Limitations and future aspirations}

There is no health without mental health. This reflects the priority for mental health. However, narrow strategies and operational bureaucracies hinder broader public health approaches for mental health and requires strategies to address these wider needs of mental health promotion of people in the country. Moreover, lack of community physicians and other health and supportive staff limits the capacity of DMH to optimally cater for mental health promotion in Sri Lanka.

While strengthening services of treatment and rehabilitation for people with mental illnesses, following the definition of 'mental health' (9) with a broader vision of making a 'Happy Sri Lanka', the DMH aims to widen its horizons to promote mental health and prevent mental health conditions among people in the country in collaboration with all relevant key stakeholders. Hence, expansion of the capacity of DMH needs to be prioritized. Furthermore, futuristic preparedness planning for MHPSS in any disaster from the lessons learnt during COVID-19 would certainly enrich holistic mental health services in Sri Lanka, making a post COVID growth and resilience in the country.

\section{Author Declaration}

Acknowledgements: We wish to acknowledge the DDG/NCD, DDG/PHS-II, College of Psychiatrists, Health Promotion Bureau, College of Community Physicians of Sri Lanka, Professor Piyanjali de Zoysa 
of Faculty of Medicine, Colombo, Dr Nisal Fernando of Ministry of Health, Media and primary health care staff.

Author contributions: All authors were involved in planning of the article and in literature search. EPW drafted the manuscript and all authors were involved in editing it. RR did the overall supervision. All authors approved the final manuscript.

\section{References}

1. Brooks SK, Webster RK, Smith LE, Woodland L, Wessely S, Greenberg N, Rubin GJ. The psychological impact of quarantine and how to reduce it: rapid review of the evidence. Lancet 2020; 395: 912-920. DOI: 10.1016/S0140-6736(20)304608.

2. WHO. Mental health and psychosocial considerations during the COVID-19 outbreak. Geneva: World Health Organization, 2020. Available from: https://www.who.int/ publications/ i/item/mental-health-and-psychosocial-considera tions-during-the-covid-19-outbreak. Accessed 30 March 2020.

3. IASC. MHPSS in Emergency Settings: Interim Briefing Note. Inter-Agency Standing Committee Reference Group 2020 (Circulated on 17 March 2020).
4. Ellepola A \& Rajapakse RPSS. Foreseeable psychological impact of COVID-19 in Sri Lanka. ResearchGate, 2020. Available from: https:// www.researchgate.net/publication/340522703. DOI: 10.13140/RG.2.2.26156.21120.

5. Rajkumar RP. COVID-19 and mental health: A review of the existing literature. Asian Journal of Psychiatry 2020: 52. DOI: 10.1016/j .ajp.2020.1 02066.

6. IASC. Inter-Agency Standing Committee Guideline on Mental Health and Psychosocial Support in Emergency Settings. Geneva: Inter-Agency Standing Committee (IASC), 2007. Available from: http://www.humanitarianinfo. org/ iasc/mental health_psychosocial_support.

7. Ministry of Health. The Mental Health Policy of Sri Lanka (2005-2015). Available from: http:/ /www.health.gov.lk./

8. Ellepola A. COVID-19 contingency plan, Teaching Hospital Anuradhapura, Sri Lanka. ResearchGate 2020. Available from:https:// www.research gate.net/publication/340582981.DOI: 10.13140/ RG.2.2.22001.22881.

9. WHO. Definition of mental health. Geneva: World Health Organization, 2004. Available from: https://www.who.int/mental_health/en. 